Introduction Interleukin (IL)-18 is a pro-inflammatory cytokine, the activity of which is regulated by its natural inhibitor IL-18 binding protein (IL-18BP). If the balance between IL-18 and IL-18BP is dysregulated, abnormal levels of free bioactive IL-18 (fIL-18) are detected, such as in the sera of patients with macrophage activation syndrome (MAS). We showed that endogenous IL-18BP exerts a protective role in a murine model of MAS induced by repeated injections of the TLR9 agonist CpG. IL-18BP production is strongly increased in liver, lung, and spleen in this model, but its cellular origin is unknown.

Objectives To study the relative contribution of radioresistant and radiosensitive cells to IL-18BP production using bone marrow (BM) transfer experiments.

Methods Following whole-body irradiation, WT or $I l 18 b p^{-1}$ recipient mice were reconstituted with WT or $I l 18 b p^{-/-} \mathrm{BM}$ to create 4 groups of mice: WT recipient mice transferred with

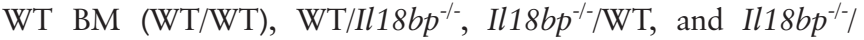
$I l 18 b p^{-/}$. BM chimeric mice were challenged with CpG injections on days 0,2 and 4 and sacrificed on day 7. We assessed body weight during the course of the experiment, blood cell counts before and after $\mathrm{CpG}$ injections and spleen weight after sacrifice. Liver, lung and spleen mRNA levels of Il18, Il18bp, Ifng, and IFN- $\gamma$ signature genes Cxcl9 and Ciita were determined by RT-qPCR. Circulating levels of IL-18BP, fIL-18, and CXCL9 were measured by ELISA.

Results The severity of CpG-induced MAS, as assessed by body weight changes, spleen weight and blood cell counts, was increased in Il18b $p^{-/ /} / I l 18 b p^{-/-}$mice, but not significantly different between the three other groups. As reflected by a higher disease severity in $I l 18 b p^{-/ /} / I l 18 b p^{-/-}$mice, an enhanced IFN- $\gamma$ signature and elevated levels of circulating fIL-18 were observed in these mice. In the other groups, IL-18BP levels were still sufficient to inhibit IL-18 activity. Indeed, circulating IL-18BP levels dropped drastically only in the $I l 18 b p^{-/} / I l 18 b p^{-/-}$group. Despite this, Il18bp $m R N A$ levels, as assessed by RT-qPCR, varied in the different organs, consistent with the relative contribution of radioresistant versus radiosensitive cells. Indeed, following $\mathrm{CpG}$ stimulation radioresistant cells were the main contributors in liver (65\%) and lung (90\%), whereas radiosensitive cells were the primary source of IL-18BP in spleen (80\%). Conclusions Our results demonstrate that IL-18BP is produced by both radioresistant and radiosensitive cells and is present at high levels in the circulation to prevent the deleterious systemic effects of IL-18 in MAS.

Disclosure of Interest None declared.

\section{P158/007 PATHOLOGICAL INFLAMMATION IN EXPERIMENTAL SPONDYLOARTHRITIS IS CHARACTERIZED BY HIGH ENDOTHELIAL VENULES AND ECTOPIC LYMPHOID STRUCTURES IN THE BONE MARROW}

${ }^{1} \mathrm{MH}$ Kaaij”, ${ }^{1} \mathrm{JP}$ van Hamburg, ${ }^{2} \mathrm{~J}$ Rip, ${ }^{3,4} \mathrm{G}$ Kollias, ${ }^{1} \mathrm{DL}$ Baeten, ${ }^{5} \mathrm{M}$ Nolte, ${ }^{1} \mathrm{LM}$ van Duivenvoorde, ${ }^{1}$ SW Tas. 'Department of Rheumatology and Clinical Immunology and Department of Experimental Immunology, Amsterdam UMC, University of Amsterdam, Amsterdam; ' Department of Pulmonary Medicine, Erasmus MC, Rotterdam, Netherlands; ${ }^{3}$ Division of Immunology, Biomedical Sciences Research Center 'Alexander Fleming', Vari; ${ }^{4}$ Department of Physiology, National and Kapodistrian University of Athens, Athens, Greece; ${ }^{5}$ Department of Plasma Proteins, Sanquin Research and Landsteiner Laboratory AMC/UvA, Amsterdam, Netherlands

10.1136/annrheumdis-2018-EWRR2019.140

Career situation of first and presenting author Student for a master or a $\mathrm{PhD}$.
Introduction Tumour necrosis factor (TNF) is important in immune-mediated inflammatory diseases such as spondyloarthritis (SpA). Transmembrane (tm)TNF-transgenic (tg) mice ${ }^{1}$ that overexpress tmTNF develop SpA symptoms, including inflammation, ectopic lymphoid structures (ELS) in bone marrow (BM), bone destruction and bone formation. SpA patients have extensive angiogenesis in inflammatory and bone forming regions.

Objectives To investigate whether there is a link between pathological angiogenesis and ELS in tmTNF tg mice in the BM.

Methods Ankles, femora, tibiae, vertebrae and spleens from 6 and 12 weeks and 8 months old tmTNF tg mice and wildtype (WT) littermates ( $n=5$ per age per group) were dissected and analyzed with confocal microscopy and analyzed 12 week old tmTNF tg and WT mice $(n=4)$ with flow cytometry. To study the importance of TNF-R signaling, tmTNF tg mice lacking TNF-RI (tmTNF tgxTNF-RI ${ }^{-/}$) or TNF-RII (tmTNF tgxTNF-RII $\left.{ }^{--}\right)$(n=4 per group) were used.

Results Immunofluorescent evaluation demonstrated that BM of tmTNF tg mice contained significantly more and extensive ELS. These ELS are limited to BM of the vertebrae and ankles, and are in close proximity of $\mathrm{MECA} 79^{+}$high endothelial venules (HEVs). ELS predominantly consisted of $\mathrm{B}_{220}{ }^{+} \mathrm{B}$ cells, of which most are $\mathrm{IgD}^{+}$naive $\mathrm{B}$ cells. Preliminary flow cytometric analysis revealed a trend towards an increase in $\mathrm{IgD}^{-C D 95}{ }^{+}$germinal center $\mathrm{B}$ cells and CXCR5 ${ }^{+}$PD $-1^{+}$FoxP3 ${ }^{-}$CTLA4 ${ }^{-} \mathrm{T}$ follicular helper cells and CXCR ${ }^{+}{ }^{\mathrm{PD}}-1^{+} \mathrm{FoxP}^{+}{ }^{+} \mathrm{CTLA} 4{ }^{+}$in the vertebrae of tmTNF tg mice compared to WT littermates. Meanwhile, B cell lineages in the BM of tmTNF tg hind limbs were not altered. Furthermore, preliminary data indicates that $\mathrm{BM}$ and spleen from tmTNF tg mice contain more $\mathrm{IgA}^{+}$plasma cells compared to WT littermates. tmTNF tgxTNF- $\mathrm{RI}^{-/}$mice did not display lymphoid aggregates or HEVs in the BM, while tmTNF tgxTNF-RII ${ }^{-/}$mice did, although to a lesser extent than tmTNF tg mice.

Conclusions tmTNF overexpression in mice results in extensive ELS associated with HEVs in the BM, which is likely to be mediated through TNF-RI signaling. HEV formation may lead to persistence of inflammation in the BM which contributes to pathology.

\section{REFERENCE}

1. Alexopoulou L, et al. Eur J Immunol 1997;27(10):2588-92

Disclosure of Interest None declared

\section{P159/030 OLT1177'TM, AN ORAL NLRP3 INFLAMMASOME INHIBITOR, INHIBITS ACUTE JOINT INFLAMMATION AND CIRCULATING IL $1 \beta$ DURING GOUT FLARES IN HUMANS}

${ }^{1}$ V Klück*, ${ }^{2} \mathrm{M}$ Janssen, ${ }^{2} \mathrm{TL}$ Jansen, ${ }^{1,3} \mathrm{IW}$ Tengesdal, ${ }^{1} \mathrm{~K}$ Schraa, ${ }^{4} \mathrm{DB}$ Skouras, ${ }^{3} \mathrm{C}$ Marchetti, ${ }^{1,3}$ CA Dinarello, ${ }^{1,5}$ LA Joosten. 'Department of Internal Medicine, RadboudUMC, Nijmegen: ${ }^{2}$ Department of Rheumatology, VieCuri MC, Venlo, Netherlands; ${ }^{3}$ Department of Medicine, University of Colorado, Aurora; ${ }^{4}$ Olatec Therapeutics LLC, New York, USA; ${ }^{5}$ Department of Medical Genetics, 'Iuliu Hatieganu' University of Medicine and Pharmacy, Cluj Napoca, Romania

10.1136/annrheumdis-2018-EWRR2019.141

Career situation of first and presenting author Young investigator. 